\title{
Pain when walking: individual sensory profiles in the foot soles of torture victims - a controlled study using quantitative sensory testing
}

\author{
Karen Prip ${ }^{1,2}$, Ann L Persson ${ }^{1}$ and Bengt H Sjölund ${ }^{2,3^{*}}$
}

\begin{abstract}
Background: With quantitative sensory testing (QST) we recently found no differences in sensory function of the foot soles between groups of torture victims with or without exposure to falanga (beatings under the feet). Compared to matched controls the torture victims had hyperalgesia to deep mechano-nociceptive stimuli and hypoesthesia to non-noxious cutaneous stimuli. The purpose of the present paper was to extend the group analysis into individual sensory profiles of victims' feet to explore possible relations between external violence (torture), reported pain, sensory symptoms and QST data to help clarify the underlying mechanisms.

Methods: We employed interviews and assessments of the pain and sensory symptoms and QST by investigators blinded to whether the patients, 32 male torture victims from the Middle East, had ( $n=15)$, or had not ( $n=17)$ been exposed to falanga. Pain intensity, area and stimulus dependence were used to characterize the pain. QST included thresholds for touch, cold, warmth, cold-pain, heat-pain, deep pressure pain and wind-up to cutaneous noxious stimuli. An ethnically matched control group was available.The normality criterion, from our control group data, was set as the mean $+/-1.28 \mathrm{SD}$, thus including $80 \%$ of all values.QST data were transformed into three categories in relation to our normality range; hypoesthesia, normoesthesia or hyperesthesia/hyperalgesia.
\end{abstract}

Results: Most patients, irrespective of having been exposed to falanga or not, reported severe pain when walking. This was often associated with hyperalgesia to deep mechanical pressure. Hypoesthesia to mechanical stimuli co-occurred with numbness, burning and with deep mechanical hyperalgesia more often than not, but otherwise, a hypoesthesia to cutaneous sensory modalities did not co-occur systematically to falanga, pain or sensory symptoms.

Conclusion: In torture victims, there seem to be overriding mechanisms, manifested by hyperalgesia to pressure pain, which is usually considered a sign of centralization. In addition there was cutaneous hypoesthesia, but since there was no obvious correlation to the localization of trauma, these findings may indicate centrally evoked disturbances in sensory transmission, that is, central inhibition. We interpret these findings as a sign of changes in central sensory processing as the unifying pathological mechanism of chronic pain in these persons.

Keywords: Chronic pain, Falanga, Nerve injury, Sensitization, Torture

\footnotetext{
*Correspondence: bsjolund@health.sdu.dk

${ }^{2}$ Institute of Public Health, University of Southern Denmark, Odense, Denmark

${ }^{3}$ Beritta Gurrisgatan 21, Malmö SE 21775, Sweden

Full list of author information is available at the end of the article
} 


\section{Background}

Many victims of torture, even after extended time periods, experience pain in the feet when walking. The use of falanga (beatings on the soles of the feet) is a torture method which deliberately aims at inflicting intense pain in the feet and lower legs [1,2]. It is nowadays used systematically as a torture method especially in the Middle East and the Far East [3]. The foot pain often persists years after torture $[2,4,5]$, and contributes to severe disabilities [6], even when walking moderate distances.

In previous studies we have confirmed and extended the clinical evidence of chronic foot pain and disability in falanga victims with chronic pain in the feet $[5,6]$. Chronic pain is also common after generalized torture. Olsen at al. [7] found that more than $80 \%$ of patients referred for rehabilitation of torture sequaele reported chronic pain. Similar figures have been reported for war veterans with post-traumatic stress disorders (PTSD;[8]). In addition, Defrin et al. [9] recently found that persons with PTSD may exhibit altered sensory processing.

When examining torture victims with quantitative sensory testing (QST), we recently and remarkably found no obvious differences in sensory function of the foot soles between groups of torture victims with or without exposure to falanga [10]. This was so in spite of the fact that almost all the falanga victims had moderate to strong pain in their feet and in twice as large an area of their foot soles as those torture victims who had not been exposed to falanga. One third of the latter did not report pain in their feet at all and many reported only slight pain. On the other hand, when compared to matched controls, torture victims had hyperalgesia to deep mechano-nociceptive stimuli, irrespective of exposure to falanga or to other forms of torture. Furthermore, cutaneous sensory fibre groups except those transmitting cold and heat pain seemed to be less sensitive to external stimuli. These findings are compatible with central changes of sensory transmission [11]. Thus, there seems to be a blend of chronic pain conditions in the feet of torture victims so that analyses of means at the group level do not reveal significant differences; thus the data should be analysed individually.

The primary objective of the present paper was to extend the group analysis of the QST data from our previous study [10] into individual sensory profiles of victims' feet to explore possible relations between external violence (torture), reported pain, sensory symptoms and QST data to help clarify the underlying pain mechanisms $[5,12]$.

\section{Methods}

\section{Participants}

The patients recruited were torture victims who had been granted asylum in Denmark. They were all referred to our centre from their general practitioner, because of their long-term sequelae from various types of torture that they had been subjected to several years earlier in their homeland. The patients were screened by an assessment team (physician, psychologist, physiotherapist and social worker), supported by an interpreter, with reference to the centre's admission criteria: 1) torture victim with asylum in Denmark; 2) physical, psychological and social needs; 3) no overt psychosis; 4) no drug or alcohol abuse; and 5) available treatment capacity.

\section{Study selection criteria}

The inclusion criteria for this study were tortured male patients originating from the Middle East and speaking Arabic or Farsi (the majority of currently referred male patients during the period May 2009 - June 2010). Seventynine consecutively referred patients were identified via the electronic patient records (see Flowchart, in [10]). One senior physician (BHS) screened these medical records according to the exclusion criteria: pathological structural changes in the feet and lower legs from reasons other than falanga, for example, sustained fractures, amputations, extensive scar tissue after burning or cuttings or foreign objects embedded in the feet such as shrapnel or bullets; nerve lesions in the lower legs from causes other than falanga, for example, diabetic or alcoholic polyneuropathy and also injury to the central nervous system such as stroke or spinal fractures. Twenty-seven persons were excluded, usually due to injury to the nervous system other than from falanga.

The reasons for exclusions were: rhizopathy $(n=7)$, diabetes $(n=3)$, not mentally fit $(n=2)$, opioid medication $(n=2)$, spinal fracture $(n=1)$, arteriosclerosis in the legs $(n=1)$, hydrocephalus $(n=1)$, not been tortured but referred for having been secondarily traumatized $(n=3)$, other problems and were referred for treatment elsewhere $(n=7)$. Clinical symptoms and signs, including the typical temporal and anatomical progression of sensory symptoms of peripheral neuropathy were always sought for during the clinical history-taking and examination.

The remaining 52 patients were invited to participate in the study. At individual meetings the examiner (KP) informed them about the purpose and methods used in the study; seventeen persons turned down the offer at this stage. If the patient agreed to participate an informed consent form was signed and dates for the assessments arranged. Three persons started but dropped out during the test sessions. Thus 32 patients participated in all three sessions. All were offered compensation for travel expenses. Arabic or Farsi interpreters assisted at all sessions. The project was carefully introduced to the three interpreters involved, all of whom had long experience in interpreting for torture victims.

It was possible to recruit 14 ethnically and age matched men from the Middle East community in Copenhagen to form a control group, going through exactly the same 
QST methods as for the patients. They had a mean age of 37 years (range 21-55), had no chronic pain conditions, and were integrated and active in the Danish society. They had lived in Denmark for an average of 15.8 years (range 7-26) and all spoke Danish.

\section{Design}

The examiner (KP) and a research assistant were blinded with regard to patient history, diagnosis and whether falanga torture had occurred or not. Breaking of the blinding took place only after the completion of all examinations and data analyses of the individual patients.

\section{Procedure and measurements}

All examinations were scheduled to $3 \times 2$ hour sessions within a two-week period and took place in a quiet room (stable temperature of $22-24^{\circ} \mathrm{C}$ ) in the research department at our centre. For details of the procedures and measurement techniques, we refer the reader to our previous paper [10].

\section{Test sites}

To our knowledge only few studies have examined the arch of the foot sole with respect to normal QST values, and the available data are mostly found from the dorsum of the foot $[13,14]$. According to current standards for QST, measurements in neuropathic pain conditions should be performed in the area with the maximum pain [15] and we therefore chose the arch of the footsole under the intermediate cuneiform bone bilaterally for all sensory tests. In addition, this site has been reported to be the most sensitive in the foot sole [16].

\section{Assessments}

\section{Interview}

The patients were asked about pain in the foot soles at rest and when walking and the findings were registered on a 3 -point Likert scale $(0=$ no pain, $1=$ slight/moderate pain, and $2=$ severe pain; cf. [5]) to determine activity related changes in foot pain. From these data the victims' feet could be divided into three groups: no foot pain; stimulusindependent foot pain (pain appearing spontaneously at rest); and stimulus-evoked foot pain (pain evoked by activity, such as walking [5]). Reported sensory disturbances such as numbness, cold/burning, pricking or buzzing sensation were also registered.

\section{Pain drawings}

The patients shaded in the locations of their pain on a special foot chart (views of right and left foot soles;[17]). The shaded-in areas on the pain drawings were measured in square millimetres and calculated in percent of the total area using a commercial software programme (Quantify One; K:L:O:N:K, Denmark; [18]).

\section{Pain intensity}

Self-reported current pain intensity was assessed on a visual analogue scale (VAS; 0-100 mm; no pain=0; worst imaginable pain=100; [19]), with reference to each foot sole separately.

\section{Quantitative sensory testing in the feet}

For a detailed description of the techniques used to measure the different modalities, see our previous study [10]. Briefly, we measured mechanical detection thresholds (MDT) utilizing Semmes Weinstein monofilaments; cold detection thresholds (CDT) and warm detection thresholds (WDT), and also cold pain thresholds (CPT) and heat pain thresholds (HPT) utilising a thermal stimulator (MEDOC Inc., Israel); and deep mechano-nociceptive thresholds (pressure pain thresholds: PTT) using an electronic pressure algometer (Somedic, Höör, Sweden). In addition, temporal summation of mechano-nociceptive stimuli (wind-up pain) was examined by using the thickest Semmes-Weinstein monofilament (size 6.65;[20]). At 0.3 $\mathrm{Hz}$ the examiner applied the filament four consecutive times to the surface of the skin in the arch of each foot sole. The patients were asked to rate the pain intensity on a VAS after the $1^{\text {st }}$ and $4^{\text {th }}$ stimulus. A 5-minute pause followed. Thereafter, to produce a more intense stimulation, 10 consecutive stimuli were applied at the rate of $1.0 \mathrm{~Hz}$. The patient rated their pain after the $1^{\text {st }}$ and $10^{\text {th }}$ stimulus. If the VAS difference between the $1^{\text {st }}$ and last stimulus was positive, a temporal summation (wind-up) had occurred.

Dynamic mechanical allodynia is a painful or unpleasant sensation evoked by a cutaneous mechanical stimulus which does not normally evoke pain. To examine this phenomenon we used light strokes with a soft brush (SENSELab $^{\mathrm{TM}}$ - Brush-05; Somedic, Hörby, Sweden). Three consecutive strokes over a $60 \mathrm{~mm}$ long distance in the arch of both foot soles were applied with the brush [21], and the patient indicated if the stimulus was unpleasant or painful.

Some of the QST stimuli that may evoke a transient unpleasant sensation or pain were applied as a pre-test in another area far from the feet (the right and left forearm [PPT], or in the palm of the hand [Windup-test]) to familiarize the patients with the procedures. It was emphasized that the QST test was not an endurance test and should not evoke pain; the patients should press the stop button or indicate to the examiner at the absolute first instance of pain detection. In this way the patient was in control at all times.

\section{Statistics}

We chose to analyse the data from the right and left feet separately, since they may share common analysis mechanisms in the central nervous system, even if separate. 
Table 1 Reported pain, sensory symptoms, pain at rest, pain when walking and type of foot pain sorted by 'pain when walking' in the left foot sole of 32 torture victims (blank space=normal)

\begin{tabular}{|c|c|c|c|c|c|c|c|c|c|c|}
\hline $\begin{array}{l}\text { Patient } \\
\text { code }\end{array}$ & $\begin{array}{l}\text { Falanga and } \\
\text { No Falanga }\end{array}$ & Numbness & $\begin{array}{l}\text { Cold } \\
\text { sensation }\end{array}$ & $\begin{array}{l}\text { Burning } \\
\text { sensation }\end{array}$ & $\begin{array}{l}\text { Pricking or } \\
\text { buzzing sensation }\end{array}$ & Dysaesthesia & Allodynia & Pain at rest & $\begin{array}{l}\text { Pain when } \\
\text { walking }\end{array}$ & $\begin{array}{l}\text { Type of } \\
\text { foot pain }\end{array}$ \\
\hline 301 & Falanga & yes & yes & . & yes & yes & . & slight/moderate & severe & evoked pain \\
\hline 302 & Falanga & & yes & yes & yes & yes & yes & slight/moderate & severe & evoked pain \\
\hline 303 & Falanga & & & yes & & yes & & no & severe & evoked pain \\
\hline 304 & No Falanga & yes & yes & yes & & & & slight/moderate & severe & evoked pain \\
\hline 306 & No Falanga & yes & & yes & & yes & & slight/moderate & severe & evoked pain \\
\hline 307 & No Falanga & yes & yes & yes & yes & . & . & slight/moderate & severe & evoked pain \\
\hline 308 & No Falanga & yes & yes & yes & yes & . & . & no & severe & evoked pain \\
\hline 309 & Falanga & . & & yes & yes & . & . & slight/moderate & severe & evoked pain \\
\hline 311 & Falanga & . & yes & yes & & . & . & no & severe & evoked pain \\
\hline 312 & Falanga & . & & . & & & & no & severe & evoked pain \\
\hline 313 & No Falanga & & & . & yes & yes & yes & slight/moderate & severe & evoked pain \\
\hline 314 & No Falanga & yes & yes & yes & yes & yes & & slight/moderate & severe & evoked pain \\
\hline 316 & Falanga & yes & . & yes & yes & yes & & slight/moderate & severe & evoked pain \\
\hline 317 & No Falanga & yes & yes & yes & yes & yes & & severe & severe & independent pain \\
\hline 322 & Falanga & & . & yes & yes & & & slight/moderate & severe & evoked pain \\
\hline 324 & Falanga & yes & . & & . & yes & & slight/moderate & severe & evoked pain \\
\hline 326 & No Falanga & yes & yes & yes & yes & yes & & slight/moderate & severe & evoked pain \\
\hline 327 & Falanga & yes & & yes & yes & yes & & slight/moderate & severe & evoked pain \\
\hline 328 & Falanga & yes & yes & yes & yes & & & slight/moderate & severe & evoked pain \\
\hline 332 & No Falanga & . & yes & . & yes & . & . & slight/moderate & severe & evoked pain \\
\hline 333 & No Falanga & yes & & yes & yes & yes & . & severe & severe & independent pain \\
\hline 337 & Falanga & yes & yes & yes & yes & yes & . & slight/moderate & severe & evoked pain \\
\hline 338 & Falanga & yes & & yes & yes & . & . & slight/moderate & severe & evoked pain \\
\hline 339 & Falanga & yes & yes & yes & yes & & & severe & severe & independent pain \\
\hline 310 & No Falanga & yes & & . & & yes & yes & no & slight/moderate & evoked pain \\
\hline 319 & Falanga & & & & . & & & slight/moderate & slight/moderate & independent pain \\
\hline 330 & No Falanga & yes & . & yes & yes & yes & & no & slight/moderate & evoked pain \\
\hline 315 & No Falanga & yes & yes & yes & & & & no & no & no pain \\
\hline 323 & No Falanga & & yes & & & & & no & no & no pain \\
\hline 325 & No Falanga & & . & & & & & no & no & no pain \\
\hline 334 & No Falanga & & . & & . & & & no & no & no pain \\
\hline 335 & No Falanga & & & . & & & & no & no & no pain \\
\hline
\end{tabular}


The mean, SD, 95\%CI, median, and range were calculated for all eight QST variables. The data were analyzed using the Statistical Package for Social Sciences (SPSS) version 18, Software for Windows (SPSS, Chicago; IL; USA).

\section{Ethics}

Each participant was informed verbally about the study and was also given an information kit containing a comprehensive written description translated into their respective languages. They also received translated guidelines concerning participation in medical research issued by the Danish Ethical Committee. The assessments comply with the Helsinki II Declaration [22] and the patients could withdraw from the study at any time, without any impact on their planned rehabilitation at our clinic. The study was approved by the Research Ethics Committee in Region Copenhagen, Denmark (H-D-2009-068) and registered in the Danish Data Protection Agency.

\section{Results}

\section{General}

All 32 patients reported pain in many parts of the body. When asked about their one most painful region, neck, shoulder and low-back pain were the most common

Table 2 Categorized sensory functions from QST data (hypo, normal=blank space, HYPER; see text), current pain intensity and pain area sorted by 'pain when walking' in the left foot sole of 32 torture victims (see Table 1)

\begin{tabular}{|c|c|c|c|c|c|c|c|c|c|c|}
\hline Patient code & MDT & CDT & WDT & CPT & HPT & PPT & Wind-up $0.3 \mathrm{~Hz}$ & Wind-up $1.0 \mathrm{~Hz}$ & VAS & Pain area \\
\hline 301 & Hypo & . & . & & . & HYPER & & & & 56 \\
\hline 302 & Hypo & & . & Нypo & . & HYPER & HYPER & HYPER & 95 & 52 \\
\hline 303 & Hypo & & . & . & . & HYPER &  & & 0 & 46 \\
\hline 304 & Нypo & Hypo & Hypo & Нyро & & . & Нypo & & 52 & 58 \\
\hline 306 & Hypo & Hypo & . & . & . & HYPER & . & . & 15 & 22 \\
\hline 307 & Hypo & Missing & Missing & Missing & Missing & HYPER & . & . & 33 & 33 \\
\hline 308 & Hypo & Hypo & Нypo & Hypo & . & HYPER & Нypo & & 29 & 1 \\
\hline 309 & Hypo & & Нypo & . & . & HYPER & . & Missing & 34 & 53 \\
\hline 311 & . & & . & . & . & & . & . & 0 & 0 \\
\hline 312 & Hypo & & . & . & . & & . & . & 0 & 6 \\
\hline 313 & Нypo & Hypo & . & . & . & HYPER & . & . & 30 & 19 \\
\hline 314 & Hypo & Нypo & Нyро & Нypo & . & & . & . & 46 & 14 \\
\hline 316 & & . & & & & . & & & 50 & 36 \\
\hline 317 & Hypo & & Нypo & . & . & HYPER & . & . & 47 & 34 \\
\hline 322 & . & & . & HYPER & . & HYPER & Missing & Missing & 53 & 47 \\
\hline 324 & Hypo & Hypo & Нypo & Hypo & . & & Нypo & & 89 & 89 \\
\hline 326 & & . & & & & HYPER & & & 100 & 3 \\
\hline 327 & . & & . & HYPER & HYPER & HYPER & . & HYPER & 65 & 70 \\
\hline 328 & Hypo & & Нуро & . & . & HYPER & . & . & 47 & 74 \\
\hline 332 & . & Hypo & . & Missing & Missing & HYPER & Missing & Missing & 47 & 65 \\
\hline 333 & Hypo & Hypo & Hypo & Hypo & . & Нyро & Нypo & & 37 & 47 \\
\hline 337 & Hypo & Hypo & Нypo & . & . & & Hypo & . & 59 & 40 \\
\hline 338 & Hypo & Hypo & Нypo & r. & . & & Нypo & & 10 & 88 \\
\hline 339 & Нypo & Hypo & & . & & . & Нypo & . & 82 & 19 \\
\hline 310 & . & Нypo & Нypo & Нypo & . & HYPER & . & . & 0 & 35 \\
\hline 319 & & Hypo & HYPER & . & HYPER & HYPER & Hypo & . & 10 & 28 \\
\hline 330 & Hypo & Hypo & Нypo & & . & HYPER & Нypo & & 20 & 36 \\
\hline 315 & Hypo & Hypo & Нуро & Нypo & . & HYPER & . & . & 0 & 0 \\
\hline 323 & . & Hypo & Нypo & . & . & & . & . & 0 & 0 \\
\hline 325 & & . & & . & & . & . & . & 0 & 0 \\
\hline 334 & Hypo & & . & HYPER & . & HYPER & Hypo & . & 0 & 0 \\
\hline 335 & Hypo & & . & . & . & HYPER & Нypo & . & 0 & 0 \\
\hline
\end{tabular}


Table 3 Reported pain, sensory symptoms, pain at rest, pain when walking and type of foot pain sorted by 'PPT, pain when walking and type of foot pain' in the left foot sole of 32 torture victims (blank space=normal) (see Table 4)

\begin{tabular}{|c|c|c|c|c|c|c|c|c|c|c|}
\hline $\begin{array}{l}\text { Patient } \\
\text { code }\end{array}$ & $\begin{array}{l}\text { Falanga and } \\
\text { No Falanga }\end{array}$ & Numbness & $\begin{array}{l}\text { Cold } \\
\text { sensation }\end{array}$ & $\begin{array}{l}\text { Burning } \\
\text { sensation }\end{array}$ & $\begin{array}{l}\text { Pricking or } \\
\text { buzzing sensation }\end{array}$ & Dysaesthesia & Allodynia & Pain at rest & $\begin{array}{l}\text { Pain when } \\
\text { walking }\end{array}$ & Type \\
\hline 306 & No Falanga & yes & . & yes & . & yes & . & slight/moderate & severe & evoked pain \\
\hline 307 & No Falanga & yes & yes & yes & yes & & & slight/moderate & severe & evoked pain \\
\hline 308 & No Falanga & yes & yes & yes & yes & & & no & severe & evoked pain \\
\hline 313 & No Falanga & . & . & & yes & yes & yes & slight/moderate & severe & evoked pain \\
\hline 326 & No Falanga & yes & yes & yes & yes & yes & & slight/moderate & severe & evoked pain \\
\hline 332 & No Falanga & . & yes & . & yes & . & & slight/moderate & severe & evoked pain \\
\hline 301 & Falanga & yes & yes & . & yes & yes & . & slight/moderate & severe & evoked pain \\
\hline 302 & Falanga & . & yes & yes & yes & yes & yes & slight/moderate & severe & evoked pain \\
\hline 303 & Falanga & . & . & yes & & yes & & no & severe & evoked pain \\
\hline 309 & Falanga & . & . & yes & yes & . & & slight/moderate & severe & evoked pain \\
\hline 322 & Falanga & & & yes & yes & . & & slight/moderate & severe & evoked pain \\
\hline 327 & Falanga & yes & & yes & yes & yes & & slight/moderate & severe & evoked pain \\
\hline 328 & Falanga & yes & yes & yes & yes & & & slight/moderate & severe & evoked pain \\
\hline 317 & No Falanga & yes & yes & yes & yes & yes & & severe & severe & independent pain \\
\hline 310 & No Falanga & yes & & & & yes & yes & no & slight/moderate & evoked pain \\
\hline 330 & No Falanga & yes & & yes & yes & yes & & no & slight/moderate & evoked pain \\
\hline 319 & Falanga & & & & . & & & slight/moderate & slight/moderate & independent pain \\
\hline 315 & No Falanga & yes & yes & yes & & . & & no & no & no pain \\
\hline 334 & No Falanga & . & . & & & . & & no & no & no pain \\
\hline 335 & No Falanga & . & . & & & . & & no & no & no pain \\
\hline 304 & No Falanga & yes & yes & yes & & . & . & slight/moderate & severe & evoked pain \\
\hline 314 & No Falanga & yes & yes & yes & yes & yes & . & slight/moderate & severe & evoked pain \\
\hline 311 & Falanga & . & yes & yes & & . & . & no & severe & evoked pain \\
\hline 312 & Falanga & . & . & & & . & & no & severe & evoked pain \\
\hline 316 & Falanga & yes & & yes & yes & yes & & slight/moderate & severe & evoked pain \\
\hline 324 & Falanga & yes & & & & yes & & slight/moderate & severe & evoked pain \\
\hline 337 & Falanga & yes & yes & yes & yes & yes & & slight/moderate & severe & evoked pain \\
\hline 338 & Falanga & yes & & yes & yes & & & slight/moderate & severe & evoked pain \\
\hline 339 & Falanga & yes & yes & yes & yes & & & severe & severe & independent pain \\
\hline 323 & No Falanga & & yes & & . & & & no & no & no pain \\
\hline 325 & No Falanga & & & & & & & no & no & no pain \\
\hline 333 & No Falanga & yes & . & yes & yes & yes & & severe & severe & independent pain \\
\hline
\end{tabular}


complaints, and only two patients who had been exposed to falanga reported their pain as being most severe in the feet. Data of reported pain and sensory symptoms for all individuals are presented separately in Tables 1, 2, 3 and 4 . We have chosen to present individual data from the left feet in this paper, since we have previously demonstrated that there is no marked difference between the two sides [10].

\section{Normality criterion}

Since the results from measuring sensory modalities with QST have varying denominations, we have chosen to transform the data into three categories in relation to our matched controls: hypoesthesia, normoesthesia and hyperesthesia/hyperalgesia (Tables 2 and 4). The normality values were derived from our control group data [10], setting normality as the mean $+/-1.28 \mathrm{SD}$, thus including $80 \%$ of all values. In fact, this criterion produced normality data (see ranges in Table 5) quite close to those in the literature. Thus, Bell-Krotoski et al. [23] and Jeng et al. [16], found the normal values for MDT of the foot sole to correspond to a mean filament size of 3.61 and 3.57 respectively, equal to a target force of $0.4 \mathrm{~g}$. As regards thermal thresholds the normal values for feet are

Table 4 Categorized sensory function from QST data (hypo, normal=blank space, HYPER; see text), current pain intensity and pain area sorted by 'PPT, pain when walking and type of foot pain' in the left foot sole of 32 torture victims

\begin{tabular}{|c|c|c|c|c|c|c|c|c|c|c|}
\hline Patient code & MDT & CDT & WDT & CPT & HPT & PPT & Wind-up $0.3 \mathrm{~Hz}$ & Wind-up $1.0 \mathrm{~Hz}$ & VAS & Pain area \\
\hline 306 & Hypo & Hypo & . & & . & HYPER & & & 15 & 22 \\
\hline 307 & Hypo & Missing & Missing & Missing & Missing & HYPER & . & . & 33 & 33 \\
\hline 308 & Hypo & Hypo & Нypo & Hypo & . & HYPER & Нypo & & 29 & 1 \\
\hline 313 & Hypo & Нypo & & . & & HYPER & . & . & 30 & 19 \\
\hline 326 & . & & . & . & . & HYPER & . & . & 100 & 3 \\
\hline 332 & . & Нypo & . & Missing & Missing & HYPER & Missing & Missing & 47 & 65 \\
\hline 301 & Hypo & & . & . & . & HYPER & r. & & . & 56 \\
\hline 302 & Hypo & & . & Нypo & . & HYPER & HYPER & HYPER & 95 & 52 \\
\hline 303 & Hypo & & . & . & . & HYPER & . & . & 0 & 46 \\
\hline 309 & Hypo & & Нypo & . & . & HYPER & . & Missing & 34 & 53 \\
\hline 322 & & . & & HYPER & & HYPER & Missing & Missing & 53 & 47 \\
\hline 327 & . & & . & HYPER & HYPER & HYPER & . & HYPER & 65 & 70 \\
\hline 328 & Нyро & & Нypo & . & . & HYPER & . & . & 47 & 74 \\
\hline 317 & Hypo & & Нypo & & . & HYPER & & & 47 & 34 \\
\hline 310 & . & Hypo & Hypo & Нypo & . & HYPER & . & . & 0 & 35 \\
\hline 330 & Hypo & Hypo & Hypo & . & . & HYPER & Нypo & . & 20 & 36 \\
\hline 319 & . & Hypo & HYPER & . & HYPER & HYPER & Hypo & . & 10 & 28 \\
\hline 315 & Hypo & Нypo & Нypo & Нypo & & HYPER & & & 0 & 0 \\
\hline 334 & Hypo & & . & HYPER & . & HYPER & Нypo & . & 0 & 0 \\
\hline 335 & Hypo & & . & . & . & HYPER & Нypo & . & 0 & 0 \\
\hline 304 & Hypo & Hypo & Нypo & Нypo & . & & Hypo & & 52 & 58 \\
\hline 314 & Hypo & Нypo & Нyро & Hypo & & . & & & 46 & 14 \\
\hline 311 & . & & . & . & . & & . & . & 0 & 0 \\
\hline 312 & Hypo & & . & . & . & . & . & . & 0 & 6 \\
\hline 316 & . & & . & - & . & & 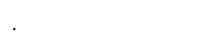 & & 50 & 36 \\
\hline 324 & Hypo & Нypo & Hypo & Нypo & . & & Hypo & & 89 & 89 \\
\hline 337 & Hypo & Hypo & Нypo & . & . & & Нypo & 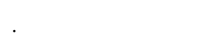 & 59 & 40 \\
\hline 338 & Нypo & Hypo & Нyро & & . & & Нyро & & 10 & 88 \\
\hline 339 & Hypo & Нypo & & . & & . & Нypo & . & 82 & 19 \\
\hline 323 & . & Hypo & Нyро & . & . & & 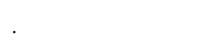 & & 0 & 0 \\
\hline 325 & . & . & & . & & . & . & . & 0 & 0 \\
\hline 333 & Hypo & Нypo & Нypo & Нypo & . & Нypo & Нypo & & 37 & 47 \\
\hline
\end{tabular}


Table 5 Sensory functions in the left foot of 14 healthy controls (mean, SD, $95 \%$ Cl, median and range for eight QST variables) and our calculated normality criteria (see text)

\begin{tabular}{|c|c|c|c|c|c|c|c|c|}
\hline & $\begin{array}{l}\text { MDT target } \\
\text { force } g\end{array}$ & $\mathrm{CDT}{ }^{\circ} \mathrm{C}$ & WDT ${ }^{\circ} \mathrm{C}$ & $\mathrm{CPT}{ }^{\circ} \mathrm{C}$ & HPT ${ }^{\circ} \mathrm{C}$ & PPT kPa & $\begin{array}{l}\text { Wind-up mm } \\
\text { at } 0.3 \mathrm{~Hz}\end{array}$ & $\begin{array}{l}\text { Wind-up mm } \\
\text { at } 1.0 \mathrm{~Hz}\end{array}$ \\
\hline & Mean & Mean & Mean & Mean & Mean & Mean & Mean & Mean \\
\hline & SD & SD & SD & SD & SD & SD & SD & SD \\
\hline & $\mathrm{Cl}$ & $\mathrm{Cl}$ & $\mathrm{Cl}$ & $\mathrm{Cl}$ & $\mathrm{Cl}$ & $\mathrm{Cl}$ & $\mathrm{Cl}$ & $\mathrm{Cl}$ \\
\hline & $\begin{array}{l}\text { Median } \\
\text { (Range) }\end{array}$ & $\begin{array}{l}\text { Median } \\
\text { (Range) }\end{array}$ & $\begin{array}{l}\text { Median } \\
\text { (Range) }\end{array}$ & $\begin{array}{l}\text { Median } \\
\text { (Range) }\end{array}$ & $\begin{array}{l}\text { Median } \\
\text { (Range) }\end{array}$ & $\begin{array}{l}\text { Median } \\
\text { (Range) }\end{array}$ & $\begin{array}{l}\text { Median } \\
\text { (Range) }\end{array}$ & $\begin{array}{l}\text { Median } \\
\text { (Range) }\end{array}$ \\
\hline \multirow[t]{5}{*}{ Controls n=14 (left foot) } & 0.33 & 28.0 & 40.9 & 21.1 & 47.0 & 456 & 17 & 27 \\
\hline & 0.34 & 2.4 & 3.1 & 4.7 & 2.5 & 167 & 13 & 22 \\
\hline & $0.14-0.53$ & $26.6-29.3$ & $39.1-42.7$ & $18.3-23.8$ & $45.5-48.4$ & $359-552$ & $10-24$ & $14-39$ \\
\hline & 0.16 & 28.9 & 41.3 & 20.6 & 47.6 & 461 & 18 & 26 \\
\hline & $(0.07-1.00)$ & $(21.6-30.2)$ & $(36.1-45.1)$ & $(9.2-27.9)$ & $(42.0-49.8)$ & $(208-783)$ & $(0-39)$ & $(-2-63)$ \\
\hline Normality criterion range & $0-0.76$ & $24.9-31.1$ & $36.9-44.9$ & $15.1-27.1$ & $43.8-50.0$ & $242-670$ & $1-34$ & $0-55$ \\
\hline
\end{tabular}

$M D T$, mechanical detection threshold; g, gram; CDT, cold detection threshold; WDT, warm detection threshold; CPT, cold pain threshold; $H P T$, heat pain threshold; $P P T$, pressure pain threshold; $\mathrm{kPa}$, kiloPascal; Wind-up $\mathrm{mm}$ at $0.3 \mathrm{~Hz}$ (mean pre/post difference in pain intensity VAS mm after wind-up stimulation at $0.3 \mathrm{~Hz}$ ); Wind-up $\mathrm{mm}$ at $1.0 \mathrm{~Hz}$ (mean pre/post difference in pain intensity VAS $\mathrm{mm}$ after wind-up stimulation at $1.0 \mathrm{~Hz}$ ).

only available from the dorsum of the foot and come from the studies of Yarnitsky et al. [24], Hagander et al. [13] and Rolke et al. [14]. For CDT the mean thresholds were $29.5^{\circ} \mathrm{C}, 31.4^{\circ} \mathrm{C}$ and $30.3^{\circ} \mathrm{C}$, respectively. Normal mean values in the literature for $\mathrm{HPT}$ in the feet are $\left(45.5^{\circ} \mathrm{C}\right.$ : Yarnitsky et al. [25]; $43.7^{\circ} \mathrm{C}$ : Hagander et al. [13]; $47.0^{\circ} \mathrm{C}$ : Rolke et al. [14]). With mechano-nociceptive stimulation, Messing et al. [26] found a mean PPT of $344 \mathrm{kPa}$ in the arch of the foot, whereas Rolke et al. [14] found a mean PPT of $572 \mathrm{kPa}$ in the dorsum of the foot. The wind-up effect elicited with $0.3 \mathrm{~Hz}$ stimuli was $15 \mathrm{~mm}$ in normal subjects (Defrin et al. [9]).

\section{Sensory profiles of pain, symptoms and QST data in the feet}

Since torture victims often present with walking difficulties, we chose to sort our patients according to how intense the reported pain in their feet was when walking (severe, slight/moderate or none; Tables 1 and 2). In the following text, all patient findings are represented by the left foot.

As can be seen in Table 1, most of our patients, irrespective of exposure to falanga, reported severe pain when walking. Such severe pain was often accompanied by sensory symptoms like burning or pricking and sometimes with dysesthesia also at rest. In Table 2 it can be seen that severe pain when walking was often associated with hyperalgesia to deep mechanical pressure (PPT) but only occasionally to current high pain intensity at rest (VAS). Hypoesthesia to mechanical stimuli (MDT) cooccurred with numbness and burning and with deep mechanical hyperalgesia (PPT) more often than not, but otherwise, a hypoesthesia to cutaneous sensory modalities did not co-occur systematically to foot trauma (falanga), pain or sensory symptoms (Tables 1 and 2). Moreover, an exaggerated wind-up reaction was rare in these patients.

\section{Re-arrangement of data to deepen analysis}

A further analysis, re-arranging the data according to: 1) the occurrence of deep hyperalgesia, 2) pain when walking and 3) type of foot pain, more clearly illustrates that this change of sensory transmission was as common after generalized torture as after falanga (Tables 3 and 4). Furthermore, out of the 20 patients with hyperalgesia to deep mechanical pressure only one patient did not have any form of cutaneous hypoesthesia. Regarding the relation to foot trauma 10/15 falanga victims and 12/17 victims of generalized torture had cutaneous mechanical hypoesthesia (MDT). Moreover, hypoesthesia to mechanical detection was associated with hypoesthesia to cold detection in 12/22 cases and with warm- and cold-detection hypoesthesia in 9/22 cases. These differences were not significant (Fisher's test).

\section{Discussion}

\section{Pain in torture victims}

From the present analysis it appears that little discriminates pain mechanisms in torture victims who have been exposed to trauma from torture on different parts of the body. Rather, it seems as if there are important overriding mechanisms, manifested by the hyperalgesia to pressure pain in the QST analysis, which is usually considered a sign of centralization [27] and is also present in fibromyalgia and whiplash associated disorders [28,29]. In addition there were frequently signs of cutaneous hypoesthesia, but since there was no obvious correlation to the localization of trauma, it may be speculated that these findings also indicate centrally evoked disturbances in sensory 
transmission, that is, central inhibition [11]. In fact, other researchers found, in a large study of Complex Regional Pain Syndromes, that a combination of sensory loss and deep mechanical hyperalgesia was present in the majority of the patients (66 -69\%; [11]). Like in their study, the large inter-individual variation of our QST findings makes it important to present data individually rather than as group means.

Regarding torture victims, our findings are on one hand similar to those of Olsen et al.[7], who found that the pain was predominantly located where the trauma had hit the body ('a local sign'); similarly, our patients who had been exposed to falanga had more intense foot pain and larger pain areas in the feet than those exposed to generalized torture. However, comparing the two tortured groups (generalized torture vs. falanga with generalized torture) we had expected that there would be substantial local differences, also in their sensory functions as examined by QST. This was not the case. Rather, we found indications that central, more than peripheral, mechanisms seem to play a critical role in these pain conditions.

Regarding the use of QST in medico-legal matters, such practice is not applicable, since the size of the effects of non-organic factors on the QST method is currently unresolved [30]. On the other hand, QST can assess both large and small fiber function as illustrated in the individual profiles.

The high side-to-side correlations of all QST parameters predict that short-term test-retest reliability within the same day should be high. However, formal determination of test-retest reliability over different time ranges of 1 day, 1 month or 1 year are not available [14].

\section{Study limitations}

The patients were refugees with residence permits in Denmark and had been referred by their general practitioner to our specialized clinic, making our sample highly selected, and thus it may not be representative of all torture victims.

With our traumatized patients, it was unfortunately not possible to collect exact information on the extent of torture. Attempts to retrieve such information may produce intense anxiety and flash backs. Moreover, all patients had been subjected to various forms of torture, increasing the risk of brain injuries [31], which may contribute to the pain reported. Torture victims are vulnerable, often forget and have difficulties in focusing attention [32]. Using QST requires cooperation from the patients and the dimensions of cognitive effects on QST findings are not resolved [30,33]. Indeed, there are recent indications that chronic widespread pain is associated with lower cognitive processing speed [34]. Furthermore, the need to use interpreters in a psychophysical study, especially in a population with high levels of anxiety and depression, may present certain problems [35].

Since many of these patients have been imprisoned for long time periods, it is important to distinguish the present findings from those elicited by peripheral neuropathy, whether from toxic, nutritional or infectious causes. However, the victims were carefully examined for such comorbidities during the medical assessment (see Methods) and secondly, motor deficits, typical for severe polyneuropathies, were never found among the included patients. Furthermore, the combination often seen in our patients, cutaneous hypoesthesia, normal nociceptive transmission and deep mechanical hyperalgesia, is not typical for peripheral neuropathies but has been reported without signs of nerve injury in, for example, chronic regional pain syndrome [11; Appendix A in 27]. However it is difficult to completely exclude the possibility of nerve injury here.

For patients with neuropathic pain, some of the QST procedures may be briefly painful, for example the Windup test. In our study design, we have considered this problem, and as is obvious from our methods, the patients were always in control. In fact, we only had to discontinue one of the sensory examination sessions due to pain.

\section{Conclusion}

In conclusion, by comparing QST data from the foot soles of 32 torture victims in chronic pain to ethnically matched healthy persons, we found hyperalgesia to deep mechanical pressure in combination with cutaneous hypoesthesia to non-nociceptive modalities to be a common sensory disturbance. We interpret these findings as a sign of changes in central sensory processing which may be the unifying pathological mechanism of chronic pain in these persons.

\section{Competing interests}

The authors declare that they have no competing interests.

\section{Authors' contributions}

KP has been active in all aspects of the study: conception, design, acquisition of data (main responsibility), analysis and interpretation of data, writing of the manuscript and final approval. ALP contributed to the conception, design, analysis, and interpretation of data (main responsibility), writing of manuscript and final approval. BHS had main responsibility for the conception and design of the study, contributed to the interpretation of data and participated in writing of manuscript and final approval.

\section{Authors' information}

$\mathrm{KP}, \mathrm{PT}, \mathrm{MSc}$, has long experience of treating victims of torture since more than twenty years at our centre. This research is intended to become part of her PhD - thesis. ALP, PT, PhD, is a Senior Researcher in pain and rehabilitation research at our centre and has extensive experience of multidisciplinary pain rehabilitation. BHS, MD, DMSc, Professor of Rehabilitation at the University of Southern Denmark, is a pain management physician and was till recently Director General of our centre.

\section{Acknowledgements}

We would like to thank Per-Erik Isberg, Department of Statistics, Lund University, for statistical guidance. The study was supported by grants from Foundation Juchum, Kong Christian den Tiendes Fond, Lippmann Fonden, Torkil Steenbeck's Legat, Danish Physiotherapist Association, 
Grosserer Andreas Collstrup and son Rudolf Collstrup's Mindelegat and Doctor Sofus Carl Emil Friisog Hustru Olga Doris Friis' Legat.

\section{Author details}

'Rehabilitation and Research Centre for Torture Victims, Copenhagen, Denmark. ${ }^{2}$ Institute of Public Health, University of Southern Denmark, Odense, Denmark. ${ }^{3}$ Beritta Gurrisgatan 21, Malmö SE 21775, Sweden.

Received: 24 May 2012 Accepted: 28 December 2012

Published: 31 December 2012

\section{References}

1. Petersen HD, Jacobsen P: Psychical and physical symptoms after torture. A prospective controlled study. Forensic Sci Int 1985, 29:179-189.

2. Rasmussen OV: Medical aspects of torture. Dan Med Bull 1990, 37(Suppl 1):1-88.

3. Rejali D: Sticks and bones. In Torture and Democracy. Oxford: Princeton University Press; 2007:273-277.

4. Forrest D: The physical after-effects of torture. Forensic Sci Int 1995, 76:77-84.

5. Prip K, Persson AL: Clinical findings in men with chronic pain after falanga torture. Clin J Pain 2008, 24:135-141.

6. Prip K, Persson AL, Sjölund BH: Self-reported activity in tortured refugees with long-term sequelae including pain and the impact of foot pain from falanga-a cross-sectional study. Disabil Rehabil 2011, 33:569-578.

7. Olsen DR, Montgomery E, Bøjholm S, Foldspang A: Prevalent musculoskeletal pain as a correlate of previous exposure to torture. Scand J Public Health 2006, 34:496-503.

8. Beckham JC, Crawford AL, Feldman ME, Kirby AC, Hertzberg MA, Davidson JR, Moore SD: Chronic posttraumatic stress disorder and chronic pain in Vietnam combat veterans. J Psychosom Res 1997, 43:379-389.

9. Defrin R, Ginzburg K, Solomon Z, Polad E, Bloch M, Govezensky M, Schreiber S: Quantitative testing of pain perception in subjects with PTSDimplications for the mechanism of the coexistence between PTSD and chronic pain. Pain 2008, 138:450-459.

10. Prip K, Persson AL, Sjölund BH: Differences of sensory function in the foot soles between victims of generalised torture, victims also beaten under the feet (falanga) and healthy controls - a blinded study using quantitative sensory testing. BMC Int Health Hum Rights 2012, 12:39.

11. Gierthmuhlen J, Maier C, Baron R, Tolle T, Treede RD, Birbaumer N, Huge V, Koroschetz J, Krumova EK, Lauchart M, Maihöfner C, Richter H, Westermann A: German Research Network on Neuropathic Pain (DFNS) study group: Sensory signs in complex regional pain syndrome and peripheral nerve injury. Pain 2012, 153:765-774.

12. Amris K, Torp-Pedersen S, Rasmussen OV: Long-term consequences of falanga torture - what do we know and what do we need to know? Torture 2009, 19:33-40.

13. Hagander LG, Midani HA, Kuskowski MA, Parry GJ: Quantitative sensory testing: effect of site and skin temperature on thermal thresholds. Clin Neurophysiol 2000, 111:17-22.

14. Rolke R, Magerl W, Campbell KA, Schalber C, Caspari S, Birklein F, Treede RD: Quantitative sensory testing: a comprehensive protocol for clinical trials. Eur J Pain 2006, 10:77-88.

15. Treede RD, Jensen TS, Campbell JN, Cruccu G, Dostrovsky JO, Griffin JW, Hansson P, Hughes R, Nurmikko T, Serra J: Neuropathic pain: redefinition and a grading system for clinical and research purposes. Neurology 2008, 70:1630-1635.

16. Jeng C, Michelson J, Mizel M: Sensory thresholds of normal human feet. Foot Ankle Int 2000, 21:501-504.

17. United Nations: Istanbul Protocol; 2001. http://www.ohchr.org/Documents/ Publications/training8Rev1en.pdf.

18. Persson AL, Garametsos S, Pedersen J: Computer aided surface estimation of pain drawings - intra and interrater reliability. J Pain Res 2011, 4:135-141.

19. Scott J, Huskisson EC: Graphic representation of pain. Pain 1976, 2:175-184.

20. Defrin R, Ohry A, Blumen N, Urca G: Characterization of chronic pain and somatosensory function in spinal cord injury subjects. Pain 2001, 89:253-263.

21. Samuelsson M, Leffler AS, Hansson P: Dynamic mechanical allodynia: on the relationship between temporo-spatial stimulus parameters and evoked pain in patients with peripheral neuropathy. Pain 2005, 115:264-272.
22. World Medical Association (WMA): Declaration of Helsinki. Ethical principles for medical research involving human subjects. http://www.wma.net/en/ 30publications/10policies/b3/index.html.

23. Bell-Krotoski JA, Fess EE, Figarola JH, Hiltz D: Threshold detection and Semmes-Weinstein monofilaments. J Hand Ther 1995, 8:155-162.

24. Yarnitsky D, Sprecher E: Thermal testing: normative data and repeatability for various test algorithms. J Neurol Sci 1994, 125:39-45.

25. Yarnitsky D, Sprecher E, Zaslansky R, Hemli JA: Heat pain thresholds: normative data and repeatability. Pain 1995, 60:329-332.

26. Messing $K$, Kilbom A: Standing and very slow walking: foot pain-pressure threshold, subjective pain experience and work activity. Appl Ergon 2001, 32:81-90.

27. Maier C, Baron R, Tolle TR, Binder A, Birbaumer N, Birklein F, Gierthmuhlen J, Flor H, Geber C, Huge V, Krumova EK, Landwehrmeyer GB, Magerl W, Maihöfner C, Richter H, Rolke R, Scherens A, Schwarz A, Sommer C, Tronnier V, Uçeyler N, Valet M, Wasner G, Treede RD: Quantitative sensory testing in the German Research Network on Neuropathic Pain (DFNS): somatosensory abnormalities in 1236 patients with different neuropathic pain syndromes. Pain 2010, 150:439-450.

28. Kosek E, Ekholm J, Hansson P: Sensory dysfunction in fibromyalgia patients with implications for pathogenic mechanisms. Pain 1996 68:375-383.

29. Wallin M, Liedberg G, Börsbo B, Gerdle B: Thermal detection and pain thresholds but not pressure pain thresholds are correlated with psychological factors in women with chronic whiplash-associated pain. Clin J Pain 2012, 28:211-221.

30. Shy ME, Frohman EM, So YT, Arezzo JC, Cornblath DR, Giuliani MJ, Kincaid JC, Ochoa JL, Parry GJ, Weimer LH: Quantitative sensory testing: report of the Therapeutics and Technology Assessment Subcommittee of the American Academy of Neurology. Neurology 2003, 60:898-904.

31. Mollica RF, Lyoo IK, Chernoff MC, Bui HX, Lavelle J, Yoon SJ, Kim JE, Renshaw PF: Brain structural abnormalities and mental health sequelae in South Vietnamese ex-political detainees who survived traumatic head injury and torture. Arch Gen Psychiatry 2009, 66:1221-1232

32. Sjölund $\mathrm{BH}$, Kastrup $\mathrm{M}$, Montgomery E, Persson AL: Rehabilitating torture survivors. J Rehabil Med 2009, 41:689-696.

33. Hansson P, Backonja M, Bouhassira D: Usefulness and limitations of quantitative sensory testing: clinical and research application in neuropathic pain states. Pain 2007, 129:256-259.

34. Lee DM, Pendleton N, Tajar A, O'Neill TW, O'Connor DB, Bartfai G, Boonen S, Casanueva FF, Finn JD, Forti G, Giwercman A, Han TS, Huhtaniemi IT, Kula K, Lean ME, Punab M, Silman AJ, Vanderschueren D, Moseley CM, Wu FC, McBeth J: EMAS Study Group: Chronic widespread pain is associated with slower cognitive processing speed in middle-aged and older European men. Pain 2010, 151:30-36

35. Sjölund $\mathrm{BH}:$ RCT field manual on rehabilitation: version 1:2. Copenhagen: Rehabilitation and Research Centre for Torture Victims; 2012. http://www. dignityinstitute.org.

\section{doi:10.1186/1472-698X-12-40}

Cite this article as: Prip et al:: Pain when walking: individual sensory profiles in the foot soles of torture victims - a controlled study using quantitative sensory testing. BMC International Health and Human Rights 2012 12:40

\section{Submit your next manuscript to BioMed Central and take full advantage of:}

- Convenient online submission

- Thorough peer review

- No space constraints or color figure charges

- Immediate publication on acceptance

- Inclusion in PubMed, CAS, Scopus and Google Scholar

- Research which is freely available for redistribution 